\title{
Mortality-Reducing Effect of Rehabilitation for COPD: Observational Propensity-Matched Cohort Study Using a Nationwide Database
}

\author{
Yasuo Nakahara MD PhD, Hideo Yasunaga MD PhD, Haruhi Inokuchi MD, \\ Naoshi Ogata MD PhD, Hiromasa Horiguchi PhD, Shinya Matsuda MD PhD, \\ Kiyohide Fushimi MD PhD, and Nobuhiko Haga MD PhD
}

\begin{abstract}
BACKGROUND: In the course of therapy of patients with COPD, non-pharmacologic treatment, such as rehabilitation, plays an important role. Although some studies have provided concrete evidence of the effectiveness of rehabilitation in improving functional outcomes in subjects with COPD, evidence of its mortality-reducing effect has been insufficient. In the present study, we examined whether rehabilitation had positive effects on in-hospital mortality of subjects with COPD. METHODS: We used the Japanese Diagnosis Procedure Combination nationwide administrative claims database. This was a retrospective cohort study, and there were 18,037 eligible subjects with COPD from 1,055 hospitals. The main outcome was in-hospital mortality rates. A one-to-one propensity score matching method was used to compare hospital mortality rates after admission between rehabilitation and non-rehabilitation groups. RESULTS: A total of 3,356 pairs of subjects were selected from the rehabilitation and non-rehabilitation groups $(n=6,712)$. Subjects in the rehabilitation program showed a reduction in the odds of mortality (odds ratio $=0.80$, 95\% CI $0.65-1.00, P=.045)$. In the subgroup analyses, the rehabilitation group had a lower in-hospital mortality in the pre-obese subgroup (body mass index 25.0-29.9) than the non-rehabilitation group $(P=.02)$. Although not significant, the rehabilitation group showed a relatively low in-hospital mortality in the Hugh-Jones dyspnea scale class 5 subgroup $(P=.066)$. CONCLUSIONS: This large nationwide cohort study showed that rehabilitation indeed contributed to a reduction of in-hospital mortality. These findings underscore the importance of adopting rehabilitation as part of the treatment of COPD. Key words: COPD; rehabilitation; mortality; propensity score; retrospective cohort study; diagnosis procedure combination. [Respir Care 2016;61(11):1497-1504. (c) 2016 Daedalus Enterprises]
\end{abstract}

\section{Introduction}

COPD is a respiratory disease characterized by progressive persistent air-flow obstruction and difficulty in breath-

Drs Nakahara, Inokuchi, Ogata, and Haga are affiliated with the Department of Rehabilitation Medicine, Graduate School of Medicine, University of Tokyo, Tokyo, Japan. Dr Yasunaga is affiliated with the Department of Clinical Epidemiology and Health Economics, School of Public Health, University of Tokyo, Tokyo, Japan. Dr Horiguchi is affiliated with the Department of Clinical Data Management and Research, Clinical Research Center, National Hospital Organization Headquarters, Tokyo, Japan. Dr Matsuda is affiliated with the Department of Preventive Medicine and Community Health, School of Medicine, University of Occupational and Environmental Health, Fukuoka, Japan. Dr Fushimi is affiliated with the Department of Health Policy and Informatics, Tokyo ing, and it is associated with an increased chronic inflammatory reaction of the airways and lungs to harmful particles and gases. ${ }^{1,2}$ According to research by the World Health Organization, COPD is the fourth-leading cause of death

Medical and Dental University Graduate School of Medicine, Tokyo, Japan.

This study was funded by a Grant-in-Aid for Research on Policy Planning and Evaluation from the Ministry of Health, Labor, and Welfare, Japan (Grants H27-Policy-Designated-009 and H27-Policy-Strategy-011); by Grant-in-Aid for Scientific Research B 22390131 from the Ministry of Education, Culture, Sports, Science, and Technology; and by the Funding Program for World-Leading Innovative R\&D on Science and Technology (FIRST program) from the Council for Science and Technology Policy, Japan (Grant 0301002001001). The authors have disclosed no conflicts of interest. 


\section{Mortality-Reducing Effect of Rehabilitation in COPD}

globally, and it is estimated that it will rank third by $2020 .^{3}$ Prevention and treatment of the disease has become an important public health issue, and the economic and social burden has grown accordingly. ${ }^{4,5}$ COPD has been reported to cause a heavy financial burden. In the European Union, the direct cost associated with all respiratory diseases accounts for about $6 \%$ of the total amount of the budget dedicated to health care, $56 \%$ of which is estimated to account for COPD-related costs. ${ }^{6}$ In the United States, the direct cost of COPD to the health care system is estimated to amount to 29.5 billion dollars, and the indirect cost is estimated to amount to 20.4 billion dollars. ${ }^{7}$

Rehabilitation provides ongoing support to patients so that they can live independently. The main objectives of rehabilitation are to help restore and maintain the respiratory function of patients with disorders caused by respiratory tract diseases, as well as to enhance the quality of life and revitalize physical and mental strength as far as possible in daily life. ${ }^{8-10}$

Studies have shown that rehabilitation is effective in terms of improving functional outcomes in subjects with COPD, and it is recommended by the American College of Chest Physicians/American Association of Cardiovascular and Pulmonary Rehabilitation (ACCP/AACVPR). The Global Initiative for Chronic Obstructive Lung Disease (GOLD) guidelines also reported that rehabilitation improves exercise capacity (level A) and health-related quality of life (level A). Meanwhile, several studies on the mortality-reducing effect of rehabilitation for subjects with COPD have been reported. ${ }^{11-14}$ However, accumulation of more research data is needed. The GOLD guidelines based the mortality-reducing effect on level B evidence, and the ACCP/AACVPR guidelines concluded that there was insufficient evidence to confirm such an effect.

In the present study, we hypothesized that rehabilitation does, in fact, contribute to an improved survival rate in patients with COPD. To test this hypothesis, we conducted propensity-matched analysis with a large sample size using a nationwide in-patient database in Japan.

\section{Methods}

\section{Data Source}

This study was a retrospective cohort study using a nationwide discharge abstract and administrative claims

\footnotetext{
Correspondence: Nobuhiko Haga MD PhD, Department of Rehabilitation Medicine, Graduate School of Medicine, University of Tokyo, 7-3-1 Hongo, Bunkyo-ku, Tokyo 113-8655, Japan. E-mail: hagan-reh@h.u-tokyo.ac.jp.
}

DOI: $10.4187 /$ respcare.04652

\section{QUICK LOOK}

\section{Current knowledge}

In the course of treatment of patients with COPD, nonpharmacological treatment, such as rehabilitation, plays an important role. Although some studies have provided concrete evidence of the effectiveness of rehabilitation in improving functional outcomes in subjects with COPD, evidence of its mortality-reducing effect has been insufficient.

\section{What this paper contributes to our knowledge}

This large nationwide cohort study showed that rehabilitation indeed contributed to a reduction of in-hospital mortality. In the pre-obese subgroup, whose BMI by itself was a factor that positively contributes to reducing mortality, rehabilitation interventions led to a further decrease in the in-hospital mortality rate.

database. For the present study, we used the Japanese Diagnosis Procedure Combination (DPC) database..$^{15-17}$ All 82 university hospitals in Japan are obliged to adopt the DPC system, although adoption by community hospitals is voluntary. The database includes the following data: unique hospital identifier; patients' age and sex; diagnoses, comorbidities at admission, and complications after admission recorded in accordance with the International Classification of Diseases, 10th Revision (ICD-10) codes and as text data in Japanese; and information coded using original Japanese codes, such as the Hugh-Jones classification, smoking index, and discharge status (mortality data were collected from the discharge status listed as "death").

Complications that occurred after admission are clearly differentiated from comorbidities that were already present at admission. To optimize accuracy, the responsible physicians are obliged to record diagnoses with reference to medical charts. In 2011, 7 million patients were included in the DPC system, which accounted for approximately $45 \%$ of all in-patient admissions to acute care hospitals in Japan that year.

This study was based on a secondary analysis of the administrative claims data. The requirement for informed consent was waived because of the anonymous nature of the data. Study approval was obtained from the institutional review board at the University of Tokyo.

\section{Subject Selection and Data}

We identified subjects age $\geq 40$ y whose diagnoses at admission were COPD and allied conditions, including bronchitis (ICD-10 codes J40-J42), emphysema (J43), and 


\section{Mortality-Reducing Effect of Rehabilitation in COPD}

other types of COPD (J44), and who were discharged from an acute care hospital between July 1, 2010 and June 30, 2011. To avoid a pseudo-positive factor, we excluded severe patients who would probably have died before undergoing rehabilitation, such as those who required ventilator management at the time of admission, because they might affect excessively the mortality of the non-rehabilitation group.

Body mass index (BMI) was classified in 4 categories $\left(<18.5,18.5-24.9,25.0-29.9\right.$, and $\left.\geq 30 \mathrm{~kg} / \mathrm{m}^{2}\right)$ in accordance with the World Health Organization BMI Classification. ${ }^{18}$ The Hugh-Jones classification is a dyspnea scale that includes 5 categories: class 1 , able to work, walk, and climb up and down slopes and stairs similarly to healthy persons of the same age; class 2 , able to walk similarly to healthy persons of the same age but unable to climb up and down slopes and stairs like other healthy persons of the same age; class 3, unable to walk like healthy persons on level ground, but able to walk more than a mile $(1.6 \mathrm{~km})$ at own pace; class 4, unable to walk $50 \mathrm{~m}$ without resting; and class 5 , shortness of breath occurs when speaking or changing clothes, and usually unable to leave home. Hospital volume was defined as the number of subjects with COPD hospitalized and was categorized into 3 tertiles (low, medium, and high), with an approximately equal number of subjects in each group. On the basis of the protocol of Quan et al, ${ }^{19}$ each ICD-10 code of comorbidity was converted into points and totaled to calculate each subject's Charlson comorbidity index (CCI), which was categorized into $1,2,3$, and $\geq 4$. We also assessed in-hospital respiratory tract infections for each subject. Smoking index (pack-years) was converted to smoking status: non-smoker (smoking index $=0$ ) and current or past smoker (smoking index $\geq 1$ ).

\section{Identification of Rehabilitation}

In the health-care payment system for DPC hospitals, each subject is reimbursed for rehabilitation, and a unique procedure code for rehabilitation is recorded in the DPC database. We identified subjects who underwent rehabilitation during the hospitalization by checking this code and divided the eligible subjects into 2 groups: a usual care group and a usual care with rehabilitation group.

\section{Statistical Analyses}

With research into comparative effectiveness using retrospective observational data, treatment assignments are not random (ie, treatment selection is biased by confounding factors, including subject backgrounds). Outcomes can reflect a lack of comparability in treatment groups rather than the effects of treatment. To address this issue, we performed one-to-one propensity score matching between subjects with and without rehabilitation. ${ }^{20-22}$ The propensity score method has previously been used to reduce potential confounding caused by unbalanced covariates. This propensity-matched analysis approach allows us to construct a randomized experiment-like situation, in which the treatment groups under investigation are comparable for observed prognostic factors. The application of propensity score matching involves estimating the propensity score, followed by matching subjects according to this score and comparing the outcomes of matched subjects. To determine the propensity score, we fitted a logistic regression model for undergoing rehabilitation as a function of age, sex, BMI, Hugh-Jones classification, CCI, smoking status (smoker or non-smoker), respiratory tract infections, and hospital volume category. The $\mathrm{C}$ statistic for evaluating the goodness of fit was calculated. Each subject who underwent rehabilitation was matched with a subject who did not, with the closest estimated propensity on the logit scale being within a specified range ( $\leq .25$ of the pooled SD of the estimated logits). Fisher's exact test was used to compare the proportions between the groups. We performed multivariate logistic regression analysis to analyze the effect of rehabilitation on in-hospital mortality, with adjustment for potential confounders. The threshold for significance was $P<.05$. All statistical analyses were conducted using IBM SPSS 19.0 (IBM Corporation, Armonk, New York).

\section{Results}

\section{Subject Background}

We identified 18,037 eligible subjects from 1,055 hospitals, among whom 4,592 subjects (25.5\%) underwent rehabilitation. Using one-to-one propensity score matching, a total of 3,356 pairs of subjects were selected from the rehabilitation and non-rehabilitation groups $(n=6,712)$. The C statistic for goodness of fit was 0.624 in the propensity-matched model. The mean stay \pm SD for all 18,037 subjects was $22.8 \pm 47.1 \mathrm{~d}$, of which the rehabilitation group was $29.2 \pm 58.5 \mathrm{~d}$ and the non-rehabilitation group was $20.5 \pm 42.3 \mathrm{~d}$. Meanwhile, the mean stay for the 6,712 propensity score-matched subjects was $23.0 \pm 48.9 \mathrm{~d}$, of which the rehabilitation group was $27.2 \pm 59.3 \mathrm{~d}$ and the non-rehabilitation group was $18.9 \pm 35.2 \mathrm{~d}$. The major causes of death in all subjects who died $(n=1,593)$ were respiratory failure $(59.3 \%)$, pulmonary infection $(23.2 \%)$, infectious focus-unknown sepsis $(3.9 \%)$, heart failure $(2.2 \%)$, and stroke $(0.5 \%)$.

Table 1 shows the demographics of all of the subjects $(N=18,037)$ and the propensity score-matched subjects $(n=6,712)$. For all subjects, an unpaired $t$ test showed that the mean age was lower in the rehabilitation than in 


\section{Mortality-Reducing Effect of Rehabilitation in COPD}

Table 1. Demographic and Clinical Characteristics of All Subjects and the Propensity-Matched Subjects

\begin{tabular}{|c|c|c|c|c|c|c|c|c|c|c|}
\hline \multirow{3}{*}{ Characteristics } & \multicolumn{5}{|c|}{ All Subjects $(N=18,037)$} & \multicolumn{5}{|c|}{ Propensity-Matched Subjects $(n=6,712)$} \\
\hline & \multicolumn{2}{|c|}{$\begin{array}{c}\text { Rehabilitation } \\
\text { Group } \\
(n=4,592)\end{array}$} & \multicolumn{2}{|c|}{$\begin{array}{c}\text { Non- } \\
\text { Rehabilitation } \\
\text { Group } \\
(n=13,445)\end{array}$} & \multirow[t]{2}{*}{$P$} & \multicolumn{2}{|c|}{$\begin{array}{c}\text { Rehabilitation } \\
\text { Group } \\
(n=3,356)\end{array}$} & \multicolumn{2}{|c|}{$\begin{array}{c}\text { Non- } \\
\text { Rehabilitation } \\
\text { Group } \\
(n=3,356)\end{array}$} & \multirow[t]{2}{*}{$P$} \\
\hline & $n$ & $\%$ & $n$ & $\%$ & & $n$ & $\%$ & $n$ & $\%$ & \\
\hline Male sex & 3,844 & 77.9 & 10,471 & 83.7 & $<.001$ & 2,806 & 83.6 & 2,828 & 84.3 & .48 \\
\hline Age, y & & & & & $<.001$ & & & & & .45 \\
\hline $40-59$ & 197 & 4.3 & 739 & 5.5 & & 155 & 4.6 & 135 & 4.0 & \\
\hline $60-69$ & 729 & 15.9 & 2,005 & 14.9 & & 553 & 16.5 & 542 & 16.2 & \\
\hline $70-79$ & 1,802 & 39.2 & 4,594 & 34.2 & & 1,362 & 40.6 & 1,355 & 40.4 & \\
\hline $80-89$ & 1,678 & 36.5 & 5,120 & 38.1 & & 1,166 & 34.7 & 1,219 & 36.3 & \\
\hline$\geq 90$ & 186 & 4.1 & 987 & 7.3 & & 120 & 3.6 & 105 & 3.1 & \\
\hline Hugh-Jones classification & & & & & $<.001$ & & & & & .49 \\
\hline 1 & 261 & 6.2 & 1,165 & 10.4 & & 211 & 6.3 & 211 & 6.3 & \\
\hline 2 & 481 & 11.5 & 1,474 & 13.2 & & 388 & 11.6 & 359 & 10.7 & \\
\hline 3 & 709 & 17.0 & 1,663 & 14.9 & & 596 & 17.8 & 562 & 16.7 & \\
\hline 4 & 1,473 & 35.3 & 3,401 & 30.5 & & 1,196 & 35.6 & 1,210 & 36.1 & \\
\hline 5 & 1,254 & 30.0 & 3,461 & 31.0 & & 965 & 28.8 & 1,014 & 30.2 & \\
\hline Body mass index, $\mathrm{kg} / \mathrm{m}^{2}$ & & & & & $<.001$ & & & & & .47 \\
\hline$<18.5$ & 1,813 & 44.9 & 4,451 & 39.4 & & 1,471 & 43.8 & 1,504 & 44.8 & \\
\hline $18.5-24.9$ & 1,886 & 46.7 & 5,680 & 50.3 & & 1,596 & 47.6 & 1,595 & 47.5 & \\
\hline $25.0-29.9$ & 277 & 6.9 & 998 & 8.8 & & 238 & 7.1 & 216 & 6.4 & \\
\hline$\geq 30$ & 63 & 1.6 & 165 & 1.5 & & 51 & 1.5 & 41 & 1.2 & \\
\hline Charlson comorbidity index & & & & & $<.001$ & & & & & .65 \\
\hline 1 & 2,067 & 45.0 & 5,990 & 44.6 & & 1,522 & 45.4 & 1,555 & 46.3 & \\
\hline 2 & 1,323 & 28.8 & 3,620 & 26.9 & & 966 & 28.8 & 975 & 29.1 & \\
\hline 3 & 751 & 16.4 & 2,236 & 16.6 & & 551 & 16.4 & 533 & 15.9 & \\
\hline$>4$ & 451 & 9.8 & 1,599 & 11.9 & & 317 & 9.4 & 293 & 8.7 & \\
\hline Smoking & 2,783 & 68.1 & 6,640 & 56.8 & $<.001$ & 2,343 & 69.8 & 2,343 & 69.8 & $>.99$ \\
\hline Respiratory tract infections & 1,004 & 21.9 & 3,341 & 24.8 & $<.001$ & 672 & 20.0 & 653 & 19.5 & .56 \\
\hline Hospital volume, subjects/y & & & & & $<.001$ & & & & & .40 \\
\hline Low $(\leq 21)$ & 1,167 & 25.4 & 4,992 & 37.1 & & 849 & 25.3 & 891 & 26.5 & \\
\hline Medium (22-41) & 1,622 & 35.3 & 4,643 & 34.5 & & 1,143 & 34.1 & 1,147 & 34.2 & \\
\hline High $(\geq 42)$ & 1,803 & 39.3 & 3,810 & 28.3 & & 1,364 & 40.6 & 1,318 & 39.3 & \\
\hline
\end{tabular}

the non-rehabilitation group $(76.0 \pm 9.1 \mathrm{y}$ vs $77.0 \pm 9.9 \mathrm{y}$, $P<.001)$ and that the proportion of subjects with a HughJones classification $\geq 3$ was higher in the rehabilitation group than in the non-rehabilitation group $(82.3 \%$ vs $76.4 \%$, $P<.001)$. Smokers (68.1\% vs $56.8 \%, P<.001)$, subjects with BMI $<18.5$ (44.9\% vs $39.4 \%, P<.001)$, and highvolume hospitals $(39.3 \%$ vs $28.3 \%, P<.001)$ were more likely to undergo rehabilitation. The use of the propensity score matching method allowed us to closely balance subjects' backgrounds between the rehabilitation and nonrehabilitation groups.

\section{Mortality in the Propensity-Matched Group}

Table 2 shows the odds ratio (OR) of in-hospital mortality in the propensity-matched groups. In the treatment of COPD, rehabilitation had a positive effect in reducing in- hospital mortality by $20 \%$ (OR $=0.80,95 \%$ CI $0.65-1.00$, $P=.045)$. Other factors associated with in-hospital mortality were sex $(\mathrm{OR}=0.53,95 \% \mathrm{CI} 0.37-0.76, P=.001$ for males compared with females), BMI $(\mathrm{OR}=2.28$, 95\% CI 1.81-2.86, $P<.001$ for $\mathrm{BMI}<18.5$ and $\mathrm{OR}=$ $0.34,95 \%$ CI $0.14-0.85, P=.02$ for BMI 25.0-29.9 compared with the normal BMI 18.5-24.9 group), HughJones classification $(\mathrm{OR}=2.55,95 \%$ CI 1.02-6.38, $P=.045$ for class 4 group and $\mathrm{OR}=10.02,95 \% \mathrm{CI}$ 4.07-24.69, $P<.001$ for class 5 group compared with class 1 group), CCI (OR $=1.96,95 \%$ CI 1.46-2.64, $P<.001$ for CCI 3 and $\mathrm{OR}=2.57,95 \% \mathrm{CI} 1.85-3.57$, $P<.001$ for $\mathrm{CCI} \geq 4$ compared with the $\mathrm{CCI}=1$ group), respiratory tract infection $(\mathrm{OR}=1.66,95 \% \mathrm{CI} 1.31-2.10$, $P<.001)$ for the respiratory tract infection group compared with the non-respiratory tract infection group). Subjects in high-volume hospitals had a lower risk of in-hos- 


\section{Mortality-Reducing Effect of Rehabilitation in COPD}

Table 2. Odds Ratio of In-Hospital Mortality in the PropensityMatched Group

\begin{tabular}{|c|c|c|c|}
\hline Factors & Odds Ratio & $95 \% \mathrm{CI}$ & $P$ \\
\hline \multicolumn{4}{|l|}{ Rehabilitation } \\
\hline No & Reference & & \\
\hline Yes & 0.80 & $0.65-1.00$ & .045 \\
\hline \multicolumn{4}{|l|}{ Sex } \\
\hline Male & Reference & & \\
\hline Female & 0.53 & $0.37-0.76$ & .001 \\
\hline \multicolumn{4}{|l|}{ Age, y } \\
\hline $40-59$ & Reference & & \\
\hline $60-69$ & 0.64 & $0.27-1.52$ & .31 \\
\hline $70-79$ & 1.38 & $0.62-3.04$ & .42 \\
\hline $80-89$ & 2.16 & $0.98-4.74$ & .056 \\
\hline$\geq 90$ & 2.44 & $0.99-5.98$ & .052 \\
\hline \multicolumn{4}{|c|}{ Hugh-Jones classification } \\
\hline 1 & Reference & & \\
\hline 2 & 0.83 & $0.27-2.58$ & .75 \\
\hline 3 & 1.84 & $0.7-4.83$ & .21 \\
\hline 4 & 2.55 & $1.02-6.38$ & .045 \\
\hline 5 & 10.02 & $4.07-24.69$ & $<.001$ \\
\hline \multicolumn{4}{|c|}{ Body mass index, $\mathrm{kg} / \mathrm{m}^{2}$} \\
\hline$<18.5$ & 2.28 & $1.81-2.86$ & $<.001$ \\
\hline $18.5-24.9$ & Reference & & \\
\hline $25.0-29.9$ & 0.34 & $0.14-0.85$ & .02 \\
\hline$\geq 30$ & 1.00 & $0.3-3.31$ & $>.99$ \\
\hline \multicolumn{4}{|c|}{ Charlson comorbidity index } \\
\hline 1 & Reference & & \\
\hline 2 & 1.21 & $0.93-1.58$ & .16 \\
\hline 3 & 1.96 & $1.46-2.64$ & $<.001$ \\
\hline$\geq 4$ & 2.57 & $1.85-3.57$ & $<.001$ \\
\hline \multicolumn{4}{|c|}{ Respiratory tract infection } \\
\hline No & Reference & & \\
\hline Yes & 1.66 & $1.31-2.10$ & $<.001$ \\
\hline \multicolumn{4}{|c|}{ Hospital volume, subjects/y } \\
\hline Low $(\leq 21)$ & Reference & & \\
\hline Medium (22-41) & 0.81 & $0.62-1.05$ & .11 \\
\hline High $(\geq 42)$ & 0.73 & $0.56-0.95$ & .02 \\
\hline
\end{tabular}

pital mortality than those in low-volume hospitals $(\mathrm{OR}=0.73,95 \%$ CI $0.56-0.95, P=.02)$. Regarding age, there was a nonsignificant tendency for increased risk of in-hospital mortality with advancing age: $\mathrm{OR}=2.16$ in the group age $80-89$ y $(P=.056)$ and $\mathrm{OR}=2.44$ in the $\geq 90$-y group $(P=.052)$ compared with the group age $40-59 \mathrm{y}$.

Table 3 shows the subgroup analyses when comparing in-hospital mortality between the subjects with and without rehabilitation stratified by the BMI categories. The rehabilitation group had a lower in-hospital mortality in the pre-obese subgroup (BMI 25.0-29.9) than the nonrehabilitation group $(P=.02)$. Although not significant, the rehabilitation group had relatively low in-hospital mortality in the underweight subgroup (BMI $<18.5, P=.062$ ).

Table 4 shows the subgroup analyses when comparing in-hospital mortality between the subjects with and without rehabilitation stratified by Hugh-Jones classification. Although not significant, the rehabilitation group showed relatively low in-hospital mortality compared with the nonrehabilitation group in the Hugh-Jones class 5 subgroup $(P=.066)$.

\section{Discussion}

We examined whether rehabilitation had a positive effect on patient in-hospital mortality in the treatment of COPD. The results showed that rehabilitation does indeed contribute to a reduction of in-hospital mortality. These findings underscore the importance of adopting rehabilitation as part of the treatment of COPD.

Based on the existing evidence, ${ }^{23}$ pharmacologic treatment is routinely performed for patients with COPD. Meanwhile, non-pharmacologic treatments, including rehabilitation, have not yet been widely adopted despite its recommendations by the ACCP/AACVPR and GOLD guidelines. According to the European Respiratory Society (ERS) COPD Audit performed in 13 European countries, $30 \%$ of the patients with COPD received rehabilitation. ${ }^{24}$ In this study, we found out that only $25.5 \%$ of patients in Japan underwent rehabilitation.

Although the guidelines present concrete evidence of the effectiveness of rehabilitation in improving functional outcomes in patients with COPD, its mortality-reducing effect remained uncertain. The GOLD guidelines evaluate rehabilitation in COPD as level $\mathrm{B}$ evidence, and the ACCP/AACVPR guidelines indicate that it does not necessarily have an effect on the survival rate, but its evidence was insufficient; therefore, the accumulation of more research data was needed. Over the past years, several studies on the mortality-reducing effect of rehabilitation for patients with COPD have been reported. ${ }^{11-14}$ The Cochrane review by Puhan et al, ${ }^{11} 3$ studies involving 110 subjects (30-40 subjects each), concluded that treatment with rehabilitation led to a reduction in the odds of death $(\mathrm{OR}=0.28,95 \%$ CI $0.10-0.84)$. Using a large sample size, the present study demonstrates that rehabilitation reduced in-hospital mortality.

Examining the factors determining the prognosis of COPD is also an important matter. Smoking is the most fully studied risk factor for COPD, and other studies pertaining to age, sex, BMI and comorbidities have also been conducted in the past. Age is often mentioned as a risk factor for COPD. In terms of sex, most recent studies have shown that the prevalence of COPD and the mortality rate were higher in men than in women. However, previous reports have also shown that in developed countries, the 


\section{Mortality-Reducing Effect of Rehabilitation in COPD}

Table 3. Subgroup Analyses Comparing In-Hospital Mortality Between the Subjects With and Without Rehabilitation Stratified by Body Mass Index Categories

\begin{tabular}{|c|c|c|c|c|c|c|c|}
\hline \multirow{3}{*}{ Body mass index, $\mathrm{kg} / \mathrm{m}^{2}$} & \multicolumn{3}{|c|}{ Rehabilitation Group } & \multicolumn{3}{|c|}{ Non-Rehabilitation Group } & \multirow{3}{*}{$P$} \\
\hline & \multirow{2}{*}{$\begin{array}{c}\text { Total } \\
(n=3,356), \\
n\end{array}$} & \multicolumn{2}{|c|}{ Death $(n=177)$} & \multirow{2}{*}{$\begin{array}{c}\text { Total } \\
(n=3,356), \\
n\end{array}$} & \multicolumn{2}{|c|}{ Death $(n=222)$} & \\
\hline & & $n$ & $\%$ & & $n$ & $\%$ & \\
\hline$<18.5$ & 1,471 & 116 & 7.9 & 1,504 & 148 & 9.8 & .062 \\
\hline $18.5-24.9$ & 1,596 & 59 & 3.7 & 1,595 & 68 & 4.3 & .41 \\
\hline $25.0-29.9$ & 238 & 0 & 0.0 & 216 & 5 & 2.3 & .02 \\
\hline$\geq 30$ & 51 & 2 & 3.9 & 41 & 1 & 2.4 & $>.99$ \\
\hline
\end{tabular}

Table 4. Subgroup Analyses Comparing In-Hospital Mortality Between the Subjects With and Without Rehabilitation Stratified by the Hugh-Jones Classification

\begin{tabular}{|c|c|c|c|c|c|c|c|}
\hline \multirow{3}{*}{ Hugh-Jones classification } & \multicolumn{3}{|c|}{ Rehabilitation Group } & \multicolumn{3}{|c|}{ Non-Rehabilitation Group } & \multirow{3}{*}{$P$} \\
\hline & \multirow{2}{*}{$\begin{array}{c}\text { Total } \\
(n=3,356), \\
n\end{array}$} & \multicolumn{2}{|c|}{ Death $(n=177)$} & \multirow{2}{*}{$\begin{array}{c}\text { Total } \\
(n=3,356) \\
n\end{array}$} & \multicolumn{2}{|c|}{ Death $(n=222)$} & \\
\hline & & $n$ & $\%$ & & $n$ & $\%$ & \\
\hline 1 & 211 & 4 & 1.9 & 211 & 1 & 0.5 & .37 \\
\hline 2 & 388 & 5 & 1.3 & 359 & 3 & 0.8 & .72 \\
\hline 3 & 596 & 12 & 2.0 & 562 & 16 & 2.8 & .44 \\
\hline 4 & 1,196 & 39 & 3.3 & 1,210 & 50 & 4.1 & .28 \\
\hline 5 & 965 & 117 & 12.1 & 1,014 & 152 & 15.0 & .066 \\
\hline
\end{tabular}

prevalence of COPD showed virtually no difference between the sexes, ${ }^{25}$ which was believed to reflect changes in smoking patterns. Fatigue, underweight, and loss of appetite have often been found in patients with severe COPD. ${ }^{26}$ A report on a previous study of the BMI and the prognosis of COPD during hospitalization has shown that the underweight group had the highest rate of hospital mortality associated with exacerbations of COPD and that the mortality rate was lowest in the pre-obese group. ${ }^{27}$ Regarding comorbidities, there is an increasing awareness that most patients with COPD have comorbidities, and that they have a considerable impact on the quality of life and survival rate. ${ }^{28}$

In comparison with the previous studies, the present study also shows a relationship between in-hospital mortality and risk factors, including age, sex, BMI, severity of dyspnea, and comorbidities. Underweight subjects had a higher mortality, and most notably a lower mortality rate was found in the pre-obese group, which was similar to the results of previous studies. Regarding the severity of dyspnea, a higher mortality rate was found in subjects categorized as class 4 or higher in the Hugh-Jones classification. With respect to comorbidities, our results showed that subjects with a CCI of $\geq 3$ had an elevated mortality rate, which supports the finding that the number of comorbidities has a considerable impact on the survival rate of patients with COPD. There are other symptoms, such as depression and anxiety, that are not included in the CCI.
Previous reports have found that these comorbidities have a high prevalence in patients with COPD and increase the mortality rate. ${ }^{29-31}$ Management of these conditions should also be part of the treatment.

We performed stratified analyses based on BMI of the effect of rehabilitation on reducing the mortality rate. These analyses are the strengths and important differences in relation to other studies. The result showed that in the pre-obese subgroup, whose BMI by itself is a factor that positively contributes to reducing mortality, rehabilitation interventions led to a further decrease in the in-hospital mortality rate. Based on these results, rehabilitation is recommended for pre-obese patients with COPD. The GOLD guidelines also emphasize the fact that being underweight or overweight is a poor prognostic factor for COPD, and nutrition therapy is an important component of the program in rehabilitation. A previous study suggests that nutritional therapy alone is not a sufficient strategy, and increased calorie intake is best accompanied by rehabilitation, even helping patients without severe nutritional depletion. ${ }^{32}$ Therefore, combining the treatment with nutrition therapy and preventing patients from losing weight is a subject that deserves greater attention in treating COPD.

Several limitations of our study should be noted. First, we extracted a subjective measure of dyspnea as the HughJones classification from an existing database; however, there were no available data on the stages of COPD and the values of pulmonary function tests. Second, the results 


\section{Mortality-Reducing Effect of Rehabilitation in COPD}

of propensity score matching in this study are generalizable only among the patients in the range of propensity scores included in the paired analysis; they may not be applicable to patients who are out of this range. Propensity score methods can reduce bias in causal estimates due to observed differences between treatment groups; however, they are still subject to biases from unobserved variables, such as, in the present study, those not included in the DPC data: laboratory data, physiological data, and radiographic image data. Third, the present study consists of various types of rehabilitation programs, ranging from simple physical rehabilitation to comprehensive rehabilitation, which are not differentiated in the DPC data; therefore, details on rehabilitation programs are not identified. Fourth, the mean stay was $29.2 \mathrm{~d}$ for subjects who underwent rehabilitation during hospitalization. The GOLD guideline recommends 6 weeks as the minimum length of an effective rehabilitation program; however, because our study is based on an existing database that does not include details on rehabilitation programs, interpretation of the meaning of the stay cannot be determined. It must also be noted that the studies referenced in the GOLD guideline predominantly focus on out-patient rehabilitation, not in-patient. ${ }^{33-35}$ Meanwhile, other studies have reported the effectiveness of in-patient rehabilitation for a period of $<6$ weeks. ${ }^{36-38}$ Other limitations include the following: the data do not include whether subjects underwent rehabilitation before and/or after hospitalization; recorded diagnoses in administrative databases are less well validated than those in planned prospective cohorts or registries; the lack of data on post-discharge status precludes an evaluation of longterm outcomes; and the smoking index in the DPC data does not specify the current smoking status.

\section{Conclusions}

Although existing guidelines for COPD have noted the need to accumulate more research data on a mortalityreducing effect of pulmonary rehabilitation in patients with COPD, this large nationwide cohort study showed that rehabilitation treatment is essential for patients with COPD in improving their survival rate. These findings underscore the importance of non-pharmacologic treatments, such as rehabilitation, in providing an effective mortality-reducing treatment for COPD. Meanwhile, the burden imposed by COPD on the health-care system is large. It is important to promote an interest in research related to this highly morbid disorder so as to raise awareness of the financial burden and to improve the prevention, treatment, and management of COPD through collaboration among people involved in all aspects of health care and health policy. This study examined the effects of rehabilitation on hospitalized subjects with COPD on the basis of DPC data. It is important, however, that rehabilitation be conducted not only during hospitalization but also be continued in the long term, including at-home care as a follow-up. This study provides further evidence related to the use of rehabilitation in the treatment of COPD.

\section{REFERENCES}

1. Celli BR, MacNee W. Standards for the diagnosis and treatment of patients with COPD: a summary of the ATS/ERS position paper. Eur Respir J 2004;23(6):932-946.

2. Hay JG, Stone P, Carter J, Church S, Eyre-Brook A, Pearson MG, et al. Bronchodilator reversibility, exercise performance and breathlessness in stable chronic obstructive pulmonary disease. Eur Respir J 1992;5(6):659-664.

3. World Health Organization. World Health Report 2000. http:// www.who.int/whr/2000/en/. Accessed April 18, 2016.

4. Mathers CD, Loncar D. Projections of global mortality and burden of disease from 2002 to 2030. PLoS Med 2006;3(11):e442.

5. Global Initiative for Chronic Obstructive Lung Disease. Global strategy for the diagnosis, management, and prevention of chronic obstructive pulmonary disease. Updated 2015. http://www.goldcopd.org/uploads/ users/files/GOLD_Report_2015_Feb18.pdf.

6. European Respiratory Society. European Lung White Book: European Respiratory Society Journals. http://www.erswhitebook.org/.

7. National Heart, Lung, and Blood Institute. Morbidity \& Mortality 2012 Chart Book on Cardiovascular, Lung and Blood Diseases. US Department of Health and Human Services, Public Health Service, National Institutes of Health. 2012. http://www.nhlbi.nih.gov/files/ docs/research/2012_ChartBook.pdf.

8. Nici L, Donner C, Wouters E, Zuwallack R, Ambrosino N, Bourbeau $\mathrm{J}$, et al. American Thoracic Society/European Respiratory Society statement on pulmonary rehabilitation. Am J Respir Crit Care Med 2006;173(12):1390-1413.

9. British Thoracic Society Standards of Care Subcommittee on Pulmonary Rehabilitation. Pulmonary rehabilitation. Thorax 2001; 56(11):827-834.

10. Ries AL, Bauldoff GS, Carlin BW, Casaburi R, Emery CF, Mahler DA, et al. Pulmonary rehabilitation: Joint ACCP/AACVPR evidencebased clinical practice guidelines. Chest 2007;131(5):4S-42S.

11. Puhan MA, Gimeno-Santos E, Scharplatz M, Troosters T, Walters EH, Steurer J. Pulmonary rehabilitation following exacerbations of chronic obstructive pulmonary disease. Cochrane Database Syst Rev 2011;(10):CD005305.

12. Behnke M, Jörres RA, Kirsten D, Magnussen H. Clinical benefits of a combined hospital and home-based exercise programme over 18 months in patients with severe COPD. Monaldi Arch Chest Dis 2003;59(1):44-51.

13. Man WD, Polkey MI, Donaldson N, Gray BJ, Moxham J. Community pulmonary rehabilitation after hospitalization for acute exacerbations of chronic obstructive pulmonary disease: randomised controlled study. BMJ 2004;329(7476): 1209.

14. Troosters T, Gosselink R, Decramer M. Short- and long-term effects of outpatient rehabilitation in patients with chronic obstructive pulmonary disease: a randomized trial. Am J Med 2000;109(3):207212.

15. Matsuda S, Ishikawa KB, Kuwabara K, Fujimori K, Fushimi K, Hashimoto H. Development and use of the Japanese case-mix system. Eurohealth 2008;14:25-30.

16. Shoda N, Yasunaga H, Horiguchi H, Matsuda S, Ohe K, Kadono Y, Tanaka S. Risk factors affecting inhospital mortality after hip fracture: retrospective analysis using the Japanese Diagnosis Procedure Combination database. BMJ Open 2012;2(3):e000416. doi: 10.1136/ bmjopen-2011-000416. 


\section{Mortality-Reducing Effect of Rehabilitation in COPD}

17. Chikuda H, Yasunaga H, Horiguchi H, Takeshita K, Kawaguchi H, Matsuda S, Nakamura K. Mortality and morbidity in dialysis-dependent patients undergoing spinal surgery: analysis of a national administrative database in Japan. J Bone Joint Surg Am 2012;94(5): 433-438.

18. WHO Expert Consultation. Appropriate body-mass index for Asian populations and its implications for policy and intervention strategies. Lancet 2004;363(9403):157-163.

19. Quan H, Sundararajan V, Halfon P, Fong A, Burnand B, Luthi JC, et al. Coding algorithms for defining comorbidities in ICD-9-CM and ICD-10 administrative data. Med Care 2005;43(11):1130-1139.

20. Rosenbaum PR, Rubin DB. Constructing a control group using multivariate matched sampling methods that incorporate the propensity score. Am Stat 1985;39:33-38

21. Yasunaga H, Horiguchi H, Kuwabara K, Matsuda S, Fushimi K, Hashimoto H, Ayanian JZ. Outcomes after laparoscopic or open distal gastrectomy for early-stage gastric cancer: a propensity-matched analysis. Ann Surg 2013;257(4):640-646.

22. Ayanian JZ, Landrum MB, Guadagnoli E, Gaccione P. Specialty of ambulatory care physicians and mortality among elderly patients after myocardial infarction. N Engl J Med 2002;347(21):1678-1686.

23. Tashkin DP, Celli B, Senn S, Burkhart D, Kesten S, Menjoge S, et al. A 4-year trial of tiotropium in chronic obstructive pulmonary disease. N Engl J Med 2008;359(15):1543-1554.

24. European Respiratory Society. An International Comparison of COPD Care in Europe Results of the First European COPD Audit. 2012. http://www.ersnet.org/images/copd_audit_web_version.pdf. Accessed April 18, 2016.

25. Mannino DM, Homa DM, Akinbami LJ, Ford ES, Redd SC. Chronic obstructive pulmonary disease surveillance: United States, 19712000. MMWR Surveill Summ 2002;51(6):1-16

26. Schols AM, Slangen J, Volovics L, Wouters EF. Weight loss is a reversible factor in the prognosis of chronic obstructive pulmonary disease. Am J Respir Crit Care Med 1998;157(6 Pt 1):1791-1797.

27. Lainscak M, von Haehling S, Doehner W, Sarc I, Jeric T, Ziherl K, et al. Body mass index and prognosis in patients hospitalized with acute exacerbation of chronic obstructive pulmonary disease. J Cachexia Sarcopenia Muscle 2011;2(2):81-86.
28. Barnes PJ, Celli BR. Systemic manifestations and comorbidities of COPD. Eur Respir J 2009;33(5):1165-1185.

29. Maurer J, Rebbapragada V, Borson S, Goldstein R, Kunik ME, Yohannes AM, et al. Anxiety and depression in COPD: current understanding, unanswered questions, and research needs. Chest 2008;134(4 Suppl):43S-56S.

30. Kunik ME, Roundy K, Veazey C, Souchek J, Richardson P, Wray NP, Stanley MA. Surprisingly high prevalence of anxiety and depression in chronic breathing disorders. Chest 2005;127(4):12051211.

31. Lou P, Chen P, Zhang P, Yu J, Wang Y, Chen N, et al. Effects of smoking, depression, and anxiety on mortality in COPD patients: a prospective study. Respir Care 2014;59(1):54-61

32. Steiner MC, Barton RL, Singh SJ, Morgan MD. Nutritional enhancement of exercise performance in chronic obstructive pulmonary disease: a randomised controlled trial. Thorax 2003;58(9):745-751.

33. Finnerty JP, Keeping I, Bullough I, Jones J. The effectiveness of outpatient pulmonary rehabilitation in chronic lung disease: a randomized controlled trial. Chest 2001;119(6):1705-1710.

34. Green RH, Singh SJ, Williams J, Morgan MDL. A randomised controlled trial of four weeks versus seven weeks of pulmonary rehabilitation in chronic obstructive pulmonary disease. Thorax 2001; 56(2):143-145.

35. Behnke M, Taube C, Kirsten D, Lehnigk B, Jörres RA, Magnussen $\mathrm{H}$. Home-based exercise is capable of preserving hospital-based improvements in severe chronic obstructive pulmonary disease. Respir Med 2000;94(12):1184-1191.

36. Greulich T, Koczulla AR, Nell C, Kehr K, Vogelmeier CF, Stojanovic D, et al. Effect of a three-week inpatient rehabilitation program on 544 consecutive patients with very severe COPD: a retrospective analysis. Respiration 2015;90(4):287-292.

37. Stewart DG, Drake DF, Robertson C, Marwitz JH, Kreutzer JS, Cifu DX. Benefits of an inpatient pulmonary rehabilitation program: a prospective analysis. Arch Phys Med Rehabil 2001;82(3):347-352.

38. Clini EM, Crisafulli E, Costi S, Rossi G, Lorenzi C, Fabbri LM, Ambrosino N. Effects of early inpatient rehabilitation after acute exacerbation of COPD. Respir Med 2009;103(10):1526-1531. 